\title{
Open Extrusion of the Talus: A case report
}

\author{
by Mark A. Hardy, DPM, FACFAS ${ }^{1}$, Stella Chuida, DPM ${ }^{2}$
}

The Foot \& Ankle Journal 1 (12): 1

Open talar extrusion is an uncommon injury that the foot and ankle surgeon may encounter. Possible sequelae of this injury include arthrosis, osteomyelitis and avascular necrosis (AVN). The authors present a case involving an open lateral talar extrusion, with further discussion including mechanism of injury, anatomical considerations, incidence of AVN and perioperative concerns.

Key words: Open dislocation, talus dislocation, avascular necrosis, Hawkin's sign

Accepted: November, $2008 \quad$ Published: December, 2008

This is an Open Access article distributed under the terms of the Creative Commons Attribution License. It permits unrestricted use, distribution, and reproduction in any medium, provided the original work is properly cited. @The Foot \& Ankle Journal (www.faoj.org)

\section{Case Report}

A 41-year-old male with past medical history of hypertension presented to the emergency department after sustaining a right ankle injury while playing basketball. He reported landing on an inverted foot after attempting a 'dunk' shot during the game. He then noted exposure of bone at the lateral aspect of his ankle. He denied losing consciousness or sustaining any other injuries. He did admit to having consumed moderate amounts of alcohol prior to the injury.

At presentation, he was in moderate distress due to pain, found to be alert and oriented to person, place and time. Vital signs were stable.

Address correspondence to: Mark A. Hardy, DPM, FACFAS Kaiser Permanente Foundation Department of Podiatric Surgery 12301 Snow Road Parma, OH 44130

Email: markhardy@sbcglobal.net

\footnotetext{
${ }^{1}$ Director, Cleveland Clinic/Kaiser Permanente. Foot \& Ankle Residency Program. Director, Foot and Ankle Trauma Service. Kaiser Permanente - Ohio Region.

2 Senior Resident, Kaiser Permanente/Cleveland Clinic Foundation Residency Program, Cleveland, Ohio.
}

Examination of the right foot and ankle revealed medial dislocation of the foot on the ankle and a $15 \mathrm{~cm}$ wound to the lateral aspect of the ankle. (Figs.1 and 2) At the proximal aspect of the wound, the distal fibula, talar dome and posterolateral talar body could be visualized.

The base of the wound was beefy red and the wound margins viable. The open wound appeared relatively clean except for a sock embedded in the wound and was without debris or foreign bodies. There was no evidence of neurovascular compromise. Pedal pulses were palpable, capillary refill time was brisk and protective sensation intact. 


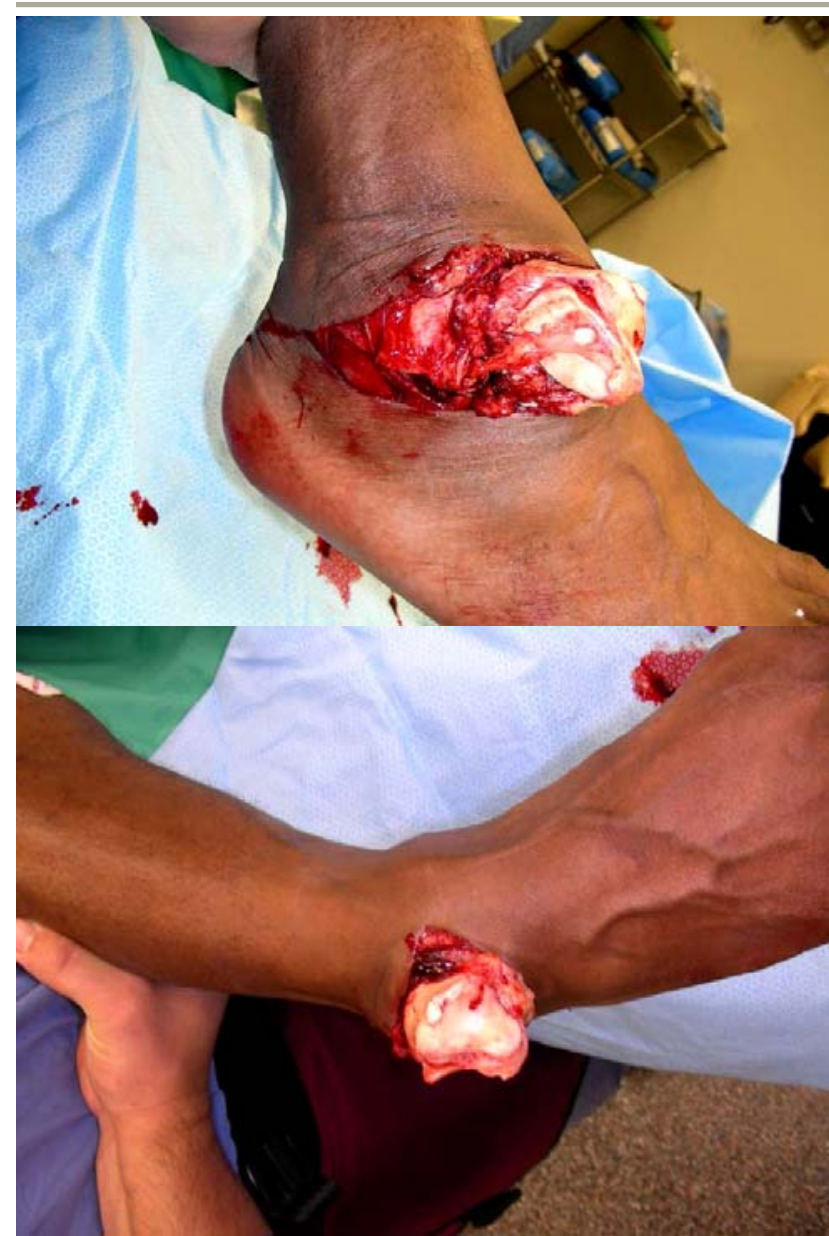

Figures 1 and 2 Gustillo-Anderson Type III open talar extrusion injury.

Radiograph of the right ankle revealed complete dislocation of the talus from the tibiotalar, subtalar and talonavicular articulations. (Fig. 3) The talus was dislocated laterally and anteriorly extruded through the skin and rotated approximately 90 degrees clockwise. The calcaneus was displaced medially and appeared to retain its normal relationship with the remaining portions of the midfoot. No definitive or occult fracture was identified. The tibia and fibula were intact and demonstrated a normal relationship, suggesting that the inferior tibiofibular ligaments were maintained. A computed tomography (CT) scan of the right ankle was also performed which confirmed the absence of any definitive fractures and the complete dislocation of the talus. (Figs. 4 and 5)

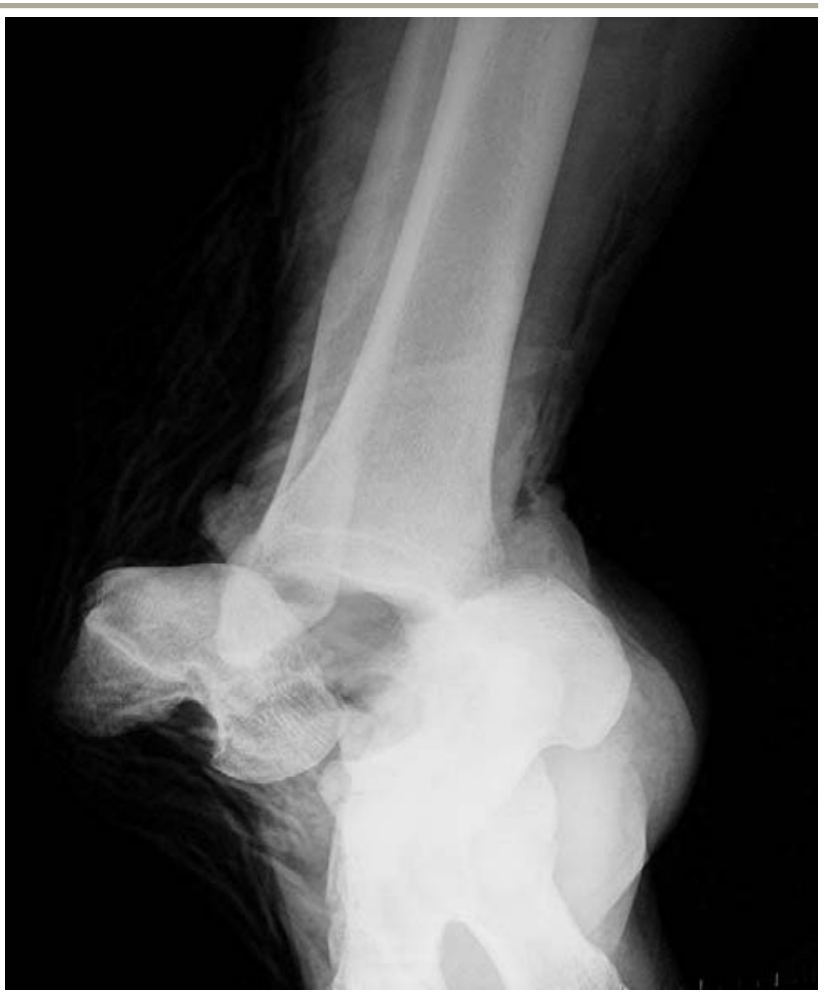

Figure 3 Plain film radiographs demonstrating talar extrusion with disruption of the tibiotalar, subtalar and talonavicular joints.

Upon admission, the patient received Ancef and Gentamycin per the senior author's open fracture protocol, in addition to ensuring tetanus prophylaxis was up to date. $\mathrm{He}$ was given intravenous Morphine for pain control. Dosing of aminoglycosides is not well defined in regards to Gustillo-Anderson type II and III open injuries ${ }^{11}$ - it is generally $3-5 \mathrm{mg} / \mathrm{kg}$ of body weight in divided doses for 3 days. However, high single dosing of aminoglycosides has been shown to be more effective than divided low dosing $(6 \mathrm{mg} / \mathrm{kg}$ given once daily). This is because the bacteriocidal effect is a result of the peak aminoglycoside concentration to minimum inhibitory concentration, not the trough levels. Moreover, nephrotoxicity and ototoxicty are a result of sustained trough concentration and not the peak concentration. 


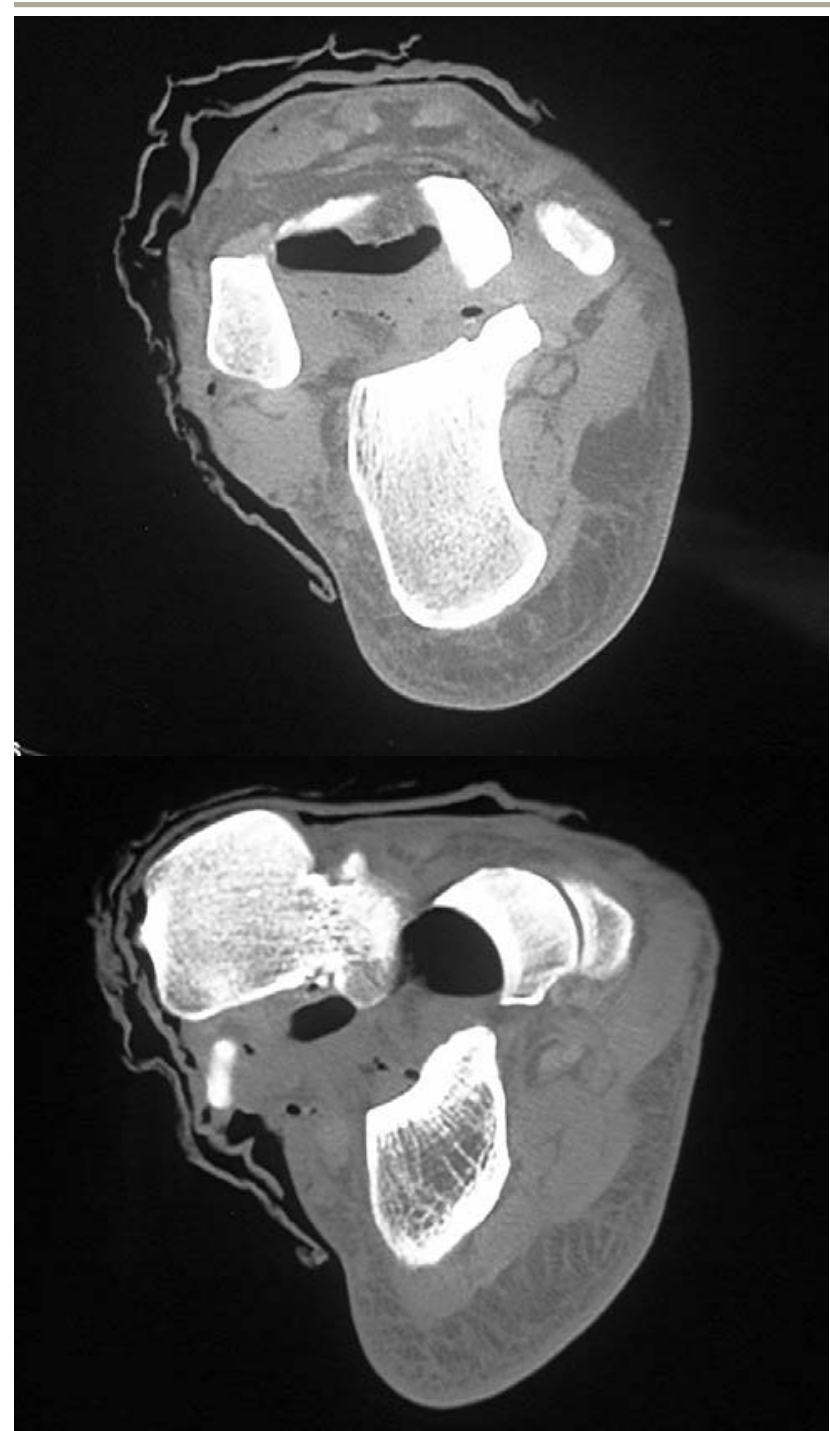

Figures 4 and 5 CT scans demonstrating the talar extrusion and absence of fracture(s).

He was taken to the operating room for emergent irrigation, debridement and relocation of the talus. The wound was irrigated with 10 liter of normal saline under pulsatile lavage and wound debridement was performed appropriately. A small osteochondral depression was noted at the lateral shoulder of the talus. This was excised along with any remaining loose cartilage. The talus was then relocated with relative ease by mild plantarflexion of the forefoot and inversion of the heel.

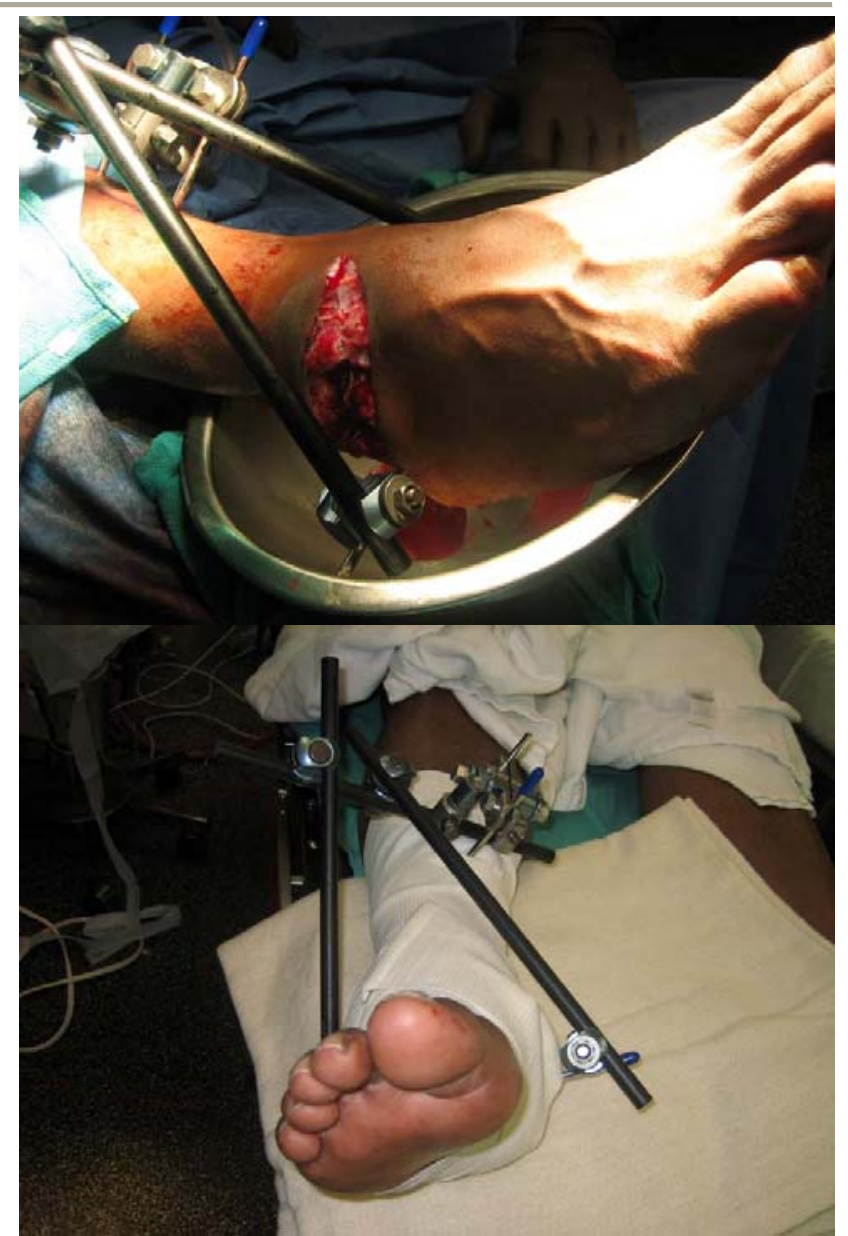

Figures 6 and 7 Intraoperative and postoperative images after reduction and stabilization with a Jet $\mathrm{X}^{\mathrm{TM}}$ (Smith and Nephew, Memphis, TN) external fixator in a simple delta frame configuration.

This was performed after the calcaneus and distal foot had been relocated under the tibia and fibula. Next, a Jet $\mathrm{X}^{\mathrm{TM}}$ (Smith and Nephew, Memphis, TN) external fixator was applied in a delta configuration to the right lower extremity. (Figs. 6 and 7)

A Steinman pin was driven from the dorsal distal navicular into the talus to maintain the reduction. (Fig. 8) The wound was then irrigated with another 6 liter of normal saline with wound cultures taken. The wound was left open with plans for delayed primary closure in approximately three days. 


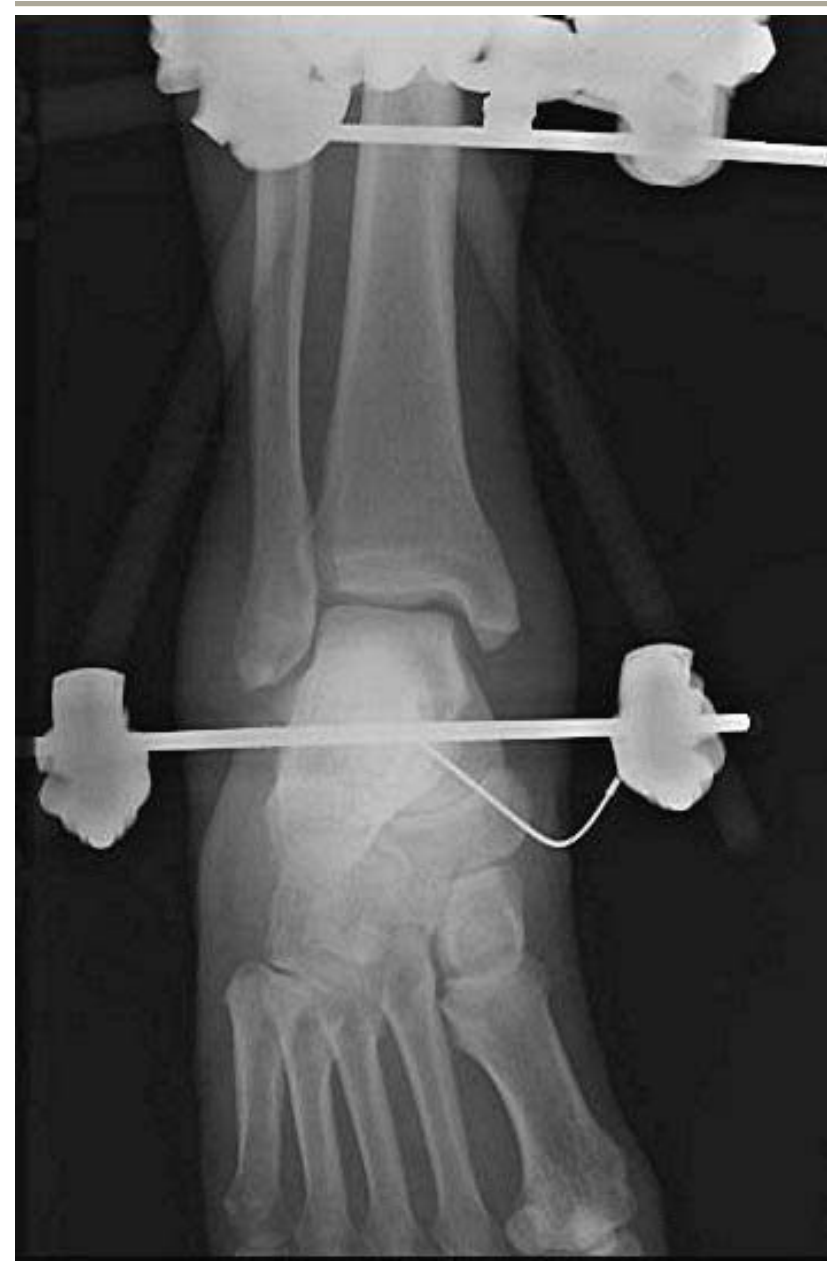

Figure 8 Postoperative radiographs after reduction and stabilization with a J et $X^{\mathrm{TM}}$ external fixator in a simple delta frame configuration. The large Steinman pin is seen through the calcaneus after reduction of the body of the talus.

The patient was admitted to the surgical floor. During his hospital stay, he had a low grade fever and mild leukocytosis during the first 24 hours, but was afebrible with a normal white count thereafter. Wound cultures were negative over three days. The wound was then closed four days later after further irrigation, at which time antibiotics were discontinued.

Postoperatively, the patient was kept non-weight bearing to the right lower extremity. The wound healed uneventfully without any signs of superficial or deep infection.

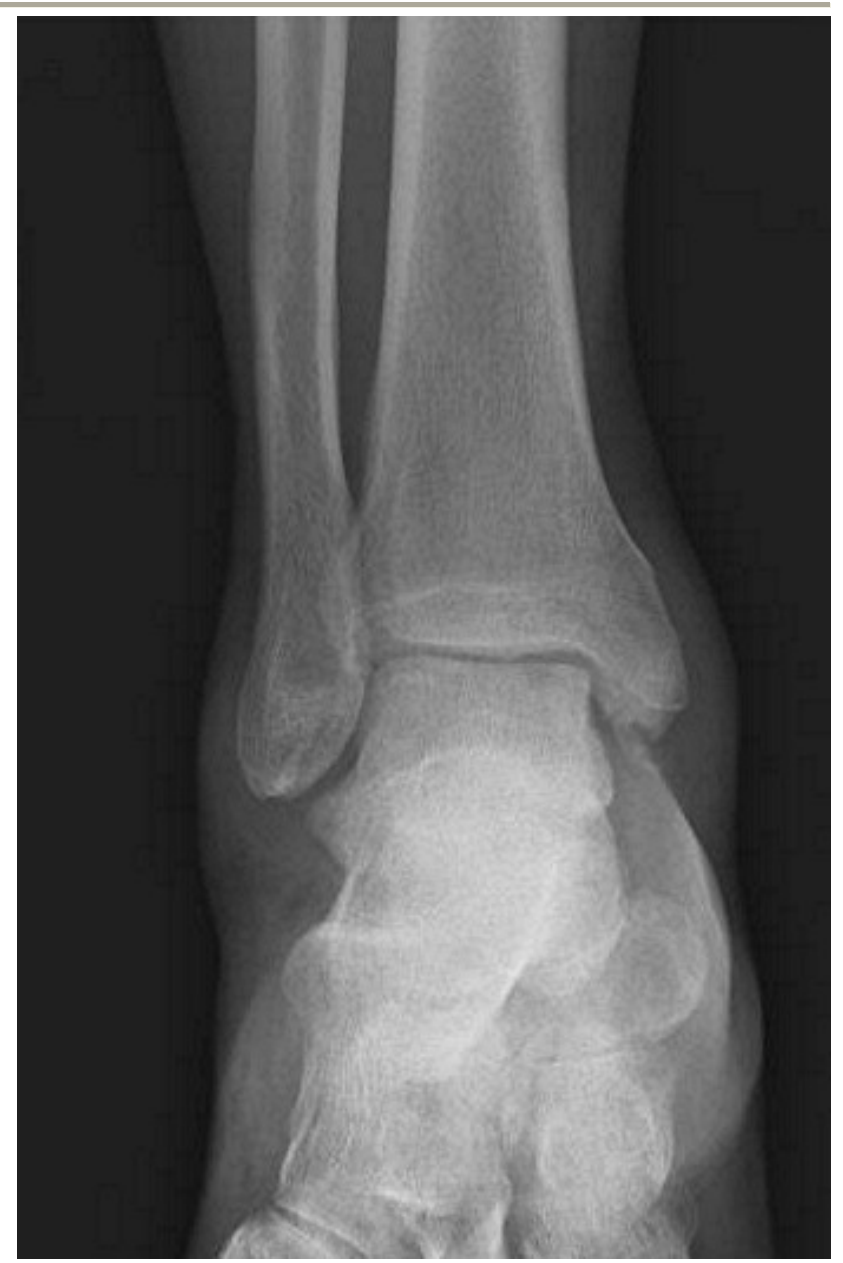

Figure 9 One month after removal of the external fixator demonstrating an early Hawkin's sign, as best seen on the mortise view.

Serial radiographs showed no evidence of osteolysis or sclerotic changes of the talus. He began using an Exogen ${ }^{\mathbf{T M}}$ bone growth stimulator (Smith and Nephew, Memphis, TN) one and half months after the initial surgery. At two months, the external fixator was removed and the patient was allowed gradual weight bearing in a fracture boot for an additional six weeks. However, he did develop superficial soft tissue infections of the transcalcaneal and distal tibial pin sites.

The calcaneal wound resolved with oral antibiotic therapy and the distal tibia pin sites healed after curettage and packing with vancomycin impregnated beads. 


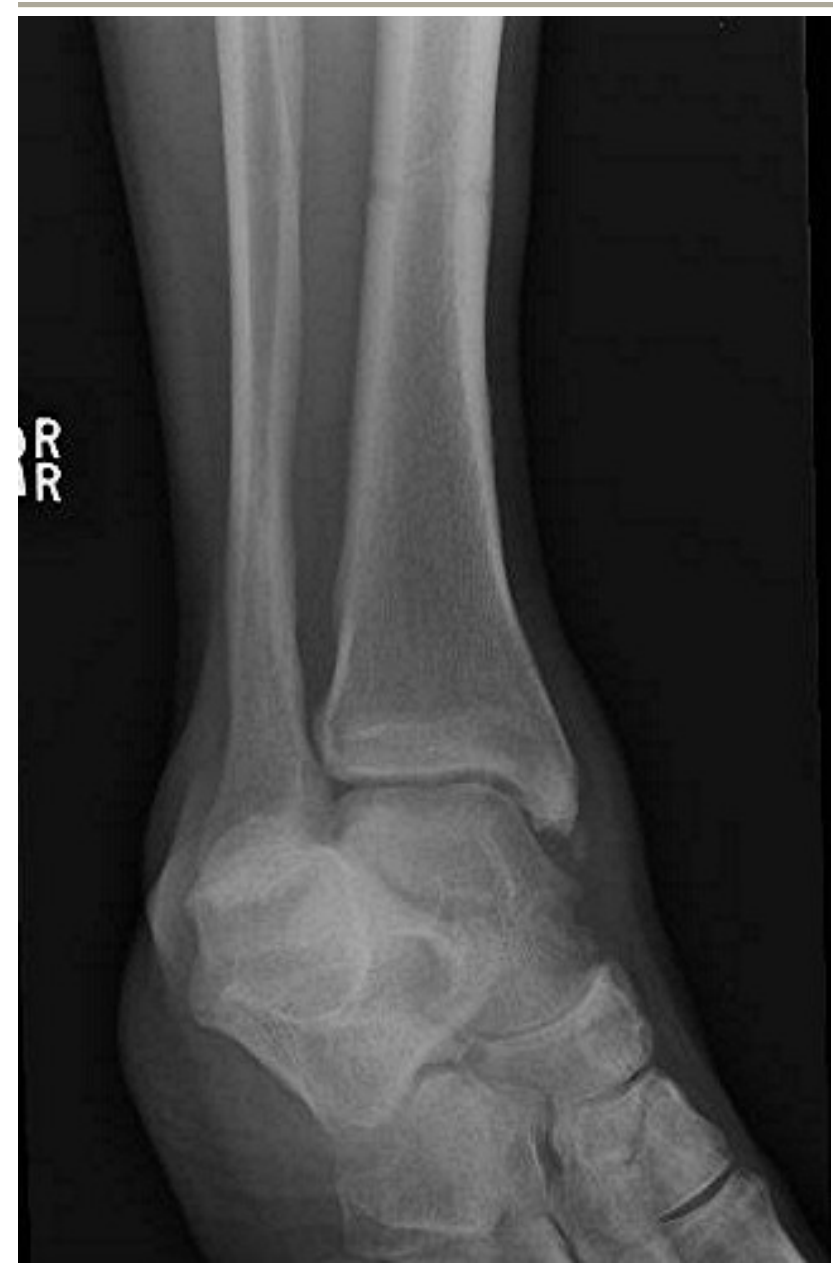

Figure 10 Another radiographic view one month after removal of the external fixator demonstrating an early Hawkins sign.

A positive Hawkins sign was noted on radiographs three months after the initial injury (Figs.9 and 10) 31/2 months postoperatively, he began ambulating in an ankle stirrup and proceeded to heal uneventfully with a full return to activities.

\section{Discussion}

According to Wagner, et al, ${ }^{1}$ the talus forms the peak of the hindfoot skeleton and transfers the whole weight of the body by itself onto the foot bones. The talus is responsible for the transfer of weight from the tibia to the foot via dissipation of forces to the subtalar, the calcaneocuboid and talonavicular joints. Three-fifths of the talus is covered by articular cartilage.
It is the only bone in the lower extremity without any muscular attachments, making it somewhat vulnerable in the presence of injury. ${ }^{2}$ Despite this, pure total dislocation without any associated fractures is rare due to the strong ligamentous attachment of the talus to adjoining midfoot bones $^{3}$ and probably due to the amount of force necessary for such an injury. ${ }^{4}$ Seventy five percent of these injuries are usually open injuries or the skin may be so tented over the talus that sloughing ultimately results. ${ }^{5}$

No specific mechanism of injury has been described. It is thought that total talar dislocation is the end point of maximum pronation or supination injuries; the final point of a continuum of forces that begin with dislocation of the subtalar joint. ${ }^{5}$ A stronger injuring force applied over a long period of time eventually forces the talus out of the mortise. Injuries can occur after falling from a height, in motor vehicle accidents or sometimes during normal physical activities.

Osteomyelitis and AVN of the talus are legitimate concerns that can affect patient morbidity when treating these injuries. Other considerations include disruption to the vascular supply of surrounding soft tissue, possible damage to lymphatics, ligaments, joint capsule and tendons; all of which increase the risk of infection of both the bone and soft tissue. The vascular supply of the talus has been well described. Although there is variation in anatomy, five major vessels supply the talus. The extraosseous vascular supply comes from the anterior tibial, the posterior tibial and the perforating peroneal arteries. The artery of the sinus tarsi is an anastamosis of the lateral tarsal artery and the perforating peroneal artery. This in turn, anastamoses with the artery of the tarsal canal, a branch of the posterior tibial artery forming an anastamotic ring about the talar neck.

The talar body is mainly supplied by the artery of the tarsal canal as well as the deltoid artery, a branch of the posterior tibial artery. ${ }^{6}$ The posterior process is supplied by small branches of the peroneal artery and calcaneal branches from the posterior tibal artery. 
The amount of articular cartilage covering the talus limits the area that remains for penetration of nutrient arteries. The likelihood of AVN is determined by the amount of soft tissue damage about the talus and how much of the numerous anastamoses as described by Mulfinger and Tureta $^{7}$ remain intact. Much of the talus is supplied by separate variable intraosseous anastamoses. Numerous anastamoses among all the arteries in the talus is one reason talar AVN is relatively uncommon. The occurrence of AVN is determined by how complete the intraosseous anastamoses are among the remaining intact vessels and the amount of damage to the soft tissue.

Treatment recommendations have evolved from primary amputation, to talectomy with tibiocalcaneal arthrodesis ${ }^{8}$ and most recently, with reinsertion of the talus. According to Huang, et al., ${ }^{9}$ reinsertion is preferable provided the wound is relatively clean and talus is still attached - even if by a soft tissue strand. Palomo-Traver, et al., ${ }^{3}$ also suggested the deformity be reduced with the exception of gross contamination or complete extrusion, as he suggested the risk of infection and AVN correlates with the degree of extrusion of the bone. Primary subtalar joint or pantalar arthrodesis has been recommended in cases of failed reduction in an effort to facilitate revascularization of the talus. Care should be taken to ensure adequate debridement of all nonviable tissue. This decreases the chances of soft tissue infection which could propagate to the bone.

In our case, the wound was not grossly contaminated with debris and there was no involvement of tendinous structures that could have tracked infection more proximally. Appropriate irrigation and debridement was performed. There was adequate soft tissue for wound closure after negative cultures; all of which helped decrease the likelihood of soft tissue or bone infection. A complete Hawkin's sign was noted at three months post injury indicating full preservation of blood supply to the talus.
A Hawkin's sign is typically seen between six to eight weeks ${ }^{10}$, but has been reported as late as two years. ${ }^{4}$ A partial Hawkins sign has also been reported which is associated with partial AVN of the talus. ${ }^{6}$ Our patient experienced none of the major complications associated with total talar dislocation. He returned to pre-injury function levels, walking and running 1-2 miles per day and reporting only occasional pain. He may not however, escape post traumatic arthritis which may develop in any or all of the joints disrupted by the injury.

\section{References}

1. Wagner, et al. Injury, Int J. Car Injured, 2004: 35, S-B36S-B45.

2. Hiraizumi Y, Hara T, Takahashi M. Mayehi S. Open total dislocation of the talus with extrusion (missing talus): Report of two cases. Foot and Ankle Int. October 13(8): 473 - 477. 1992.

3. Palomo-Traver JM, Cruz-Renovell E, Granell-Beltran V, Monzonís-García J. Open total talus dislocation: case report and review of the literature. J Ortho Trauma 11 (1): 4 5 - 49, 1997.

4. Maffulli N, Francobandiera C, Lepore L, Cifarelli V. Total dislocation of the talus. J Foot Surg, 28(3): 208-211, 1989.

5. Ries M, Healy WA Jr. Total dislocation of the talus: Case report with a 13-year follow up and review of the literature. Ortho Rev 17(1): 76-80, 1988.

6. Tehranzadeh J, Stuffman E, Ross SD. Partial Hawkins sign in fracture of the talus: a report of 3 cases. AJR Am J Roentgenol 18 (6): 1559-1563, 2003.

7. Mulfinger GL, Trueta J. The blood supply of the talus. J Bone Joint Surg 52B (1):160-7, 1970.

8. Detenberg LC, Kelly PJ. Total dislocation of the talus. JBJS 51A (2): 283-88, 1969.

9. Huang PJ, Fu YC, Tien YC, Lin GT, Lin SY et al. Open total talar dislocations - a report of 2 cases. Kaohsiung J Med Sci 16 (4): 214-218, 2000.

10. Donnelly EF. The Hawkins sign. Radiology 210 (1): 195 $-196,1999$.

11. Zalavras CG, Patzakis MJ, Holtom P. Local antibiotic therapy in the treatment of open fractures and osteomyelitis. Clin Orthop Rel Res 427: 86-93, 2004. 